\title{
Ceqmestáo
}

\section{Modelo para análise do serviço de referência virtual: uma análise quantitativa}

\author{
Ieda Pelógia Martins Damian \\ Doutora; Universidade de São Paulo (USP); São Paulo, SP, Brasil; \\ iedapm@usp.br
}

\begin{abstract}
Resumo: Com muitos recursos que ainda devem ser explorados, o serviço de referência virtual se relaciona com as maneiras de disponibilizar o serviço de referência por meio de tecnologia de informação, em benefício tanto das bibliotecas universitárias quanto dos usuários que interagem em ambiente digital. Dentro do contexto das bibliotecas universitárias, e através da análise de ferramentas desenvolvidas e utilizadas em contexto eletrônico, este estudo se propõe a desenvolver um modelo de análise do serviço de referência virtual a partir do ponto de vista de seus usuários, com o intuito de que os serviços de referência virtuais tenham seus valores realmente acrescidos. Este modelo foi utilizado e analisado junto aos usuários de serviço de referência virtual oferecidos por bibliotecas universitárias, para que desta forma pudesse ser validado e, consequentemente servir de instrumento de mensuração destes serviços.
\end{abstract}

Palavras-chave: Serviço de referência virtual. Satisfação do usuário. Serviço de referência: análise.

\section{Introdução}

Uma das mais significativas inovações dentro do campo da Tecnologia da Informação (TI) foi o surgimento e o aprimoramento da internet, que possibilita a criação, o desenvolvimento e o oferecimento de serviços de maneira inovadora. Dentre a série de vantagens oferecidas pela Internet, McNeal et al. (2003) destacou o aumento da flexibilidade no âmbito da comunicação, a redução de custos e a possibilidade de troca de grandes quantidades de informação, independentemente da distância geográfica. Estes aspectos podem ser utilizados para o oferecimento de Serviços de Referência Virtuais (SRVs) de qualidade reconhecida por seus usuários. SRV, segundo Silva e Beuttenmüller (2005), pode ser entendido como um novo recurso eletrônico que tem por finalidade atender usuários que busquem informações 
nas bibliotecas, mesmo que se encontrem fisicamente distantes.

Em 2005, Hernon e Calvert ressaltaram que apesar do importante papel desempenhado pelo ambiente digital na transformação de serviços da biblioteca em serviços eletrônicos, não existiam instrumentos de pesquisa que focassem exclusivamente na biblioteca, examinando a qualidade dos serviços prestados.

O objetivo geral deste estudo é propor um modelo para a análise dos serviços de referência virtuais partindo do ponto de vista de seus usuários, uma vez que ao analisar a literatura existente sobre SRV, é possível perceber que a maioria dos estudos relacionados à análise destes serviços em bibliotecas universitárias tradicionais está voltado para a análise de aspectos que auxiliam na avaliação do SRV do ponto de vista de infraestrutura das bibliotecas envolvidas, sem considerar os usuários destes serviços.

Silva e Beuttenmüller (2005) acreditam que estudos sobre SRVs no Brasil sejam necessários para contribuir com a consolidação do serviço que fornece a informação solicitada, utilizando os recursos eletrônicos para facilitar a resposta das questões de referência aos usuários, independente de seu formato ou meio. Diante disso, uma ferramenta de análise poderia tornar-se um importante instrumento estratégico ao sugerir soluções para aperfeiçoar a interação com os usuários das bibliotecas universitárias.

A fim de alcançar os objetivos propostos, foi realizado um levantamento bibliográfico sobre os conceitos chave para a pesquisa que são: serviço de referência virtual, modelos de avaliação de serviços de comércio eletrônico e a satisfação do usuário destes serviços. Enquanto isso, na metodologia da pesquisa, foi utilizada a pesquisa por meio de questionário com os usuários de SRVs, e os dados obtidos foram analisados com técnicas estatísticas multivariadas.

\section{Referencial teórico}

Esta seção faz uma revisão sobre serviço de referência virtual, negócios eletrônicos, satisfação do usuário com o serviço de referência virtual, qualidade e satisfação, e as considerações sobre o modelo para análise dos serviços de referência virtuais. 


\subsection{Serviço de referência virtual}

De acordo com a Reference and User Services Associantion (2015), os serviços de referência são consultas de informação que o pessoal da biblioteca recomenda, interpreta, avalia e/ou faz uso de recursos de informação para ajudar usuários a satisfazerem suas necessidades de informação. A RUSA, em 2004, também definiu o SRV como um serviço de referência iniciado em um contexto eletrônico, usualmente em tempo real, onde os usuários utilizam recursos tecnológicos para se comunicarem com os profissionais de referência, sem estarem fisicamente presentes.

Carvalho e Lucas (2005) destacam algumas vantagens competitivas do SRV, como o fato de que as fontes de informação virtual se atualizam rapidamente, demandam menor mão de obra, não necessitam de espaço físico para o armazenamento, além de que o processo de recuperação da informação tanto por parte do usuário quanto do bibliotecário torna-se otimizada e precisa. Outros benefícios do SRV também são destacados por Accart (2012), como ser acessível em todos os lugares e a todo instante, além de geralmente oferecer serviço gratuito, garantir a proteção das informações, dar orientações de fontes de informação confiáveis e oferecer um serviço personalizado e de qualidade.

Ainda que os SRVs possam oferecer uma série de benefícios, também existem pontos que precisam ser revisados. Pereira e Brenha (2011) destacaram que a montagem e a manutenção da tecnologia adequada para suportar serviço via web são dispendiosos para os orçamentos apertados das bibliotecas, ao passo que conforme Silva e Beuttenmüller (2005), a falta do bibliotecário de referência e de estratégias de marketing, sejam pontos negativos que podem inviabilizar a execução de um SRV de excelência.

Mesmo com a diversidade de serviços disponíveis na rede, Siqueira (2010) chama a atenção para o fato de que principalmente no âmbito nacional, existe, a reprodução de um modelo tradicional de assistência no balcão de referência, com profissionais da área que ainda não se adequaram a tal realidade. Arellano (2001) observou que, enquanto nos EUA há uma diversidade de SRVs mais dinâmicos, no Brasil ainda predomina a natureza mais estática dos serviços de referência. Para 
Siqueira (2010), isso é resultado de fatores como as dificuldades encontradas para se instituírem as redes colaborativas em bibliotecas e os entraves tecnológicos principalmente na esfera pública, mas principalmente a resistência ideológica e centrada numa cultura bibliográfica.

No Brasil, conforme Silva e Beuttenmüller (2005), o desenvolvimento de SRV ocorria apenas em alguns sites de bibliotecas universitárias, e que esta situação podia ter se dado devido aos subsídios para desenvolvimento do ensino e pesquisa que estas bibliotecas recebiam. Em agosto de 2009, conforme Santana et al. (2010), a Biblioteca da Faculdade de Medicina Veterinária e Zootecnia da Universidade de São Paulo (USP) implantou o serviço de atendimento online por chat, disponibilizando um novo canal de comunicação que permite o esclarecimento de dúvidas e solicitações em tempo real com um atendente. O serviço foi considerado uma iniciativa positiva e levou a implantação do sistema em outras bibliotecas da USP. Segundo os autores, no Brasil existem poucos serviços de Atendimento Online que se enquadram nos moldes do que vem sendo desenvolvido pela USP.

Com o intuito de destacar a importância do desenvolvimento do SRVs, Katz (2002) fez uma importante contribuição, especialmente para esta pesquisa, ao afirmar que as bibliotecas deveriam aprender com os sites comerciais maneiras e ferramentas utilizadas para atrair e atender seus usuários. A partir disso, o próximo item discorre sobre definições e avanços alcançados no campo dos negócios eletrônicos que podem contribuir para o desenvolvimento dos SRVs.

\subsection{Negócios eletrônicos}

Segundo Laudon e Laudon (2010), os negócios eletrônicos (e-business) podem ser definidos como a utilização das tecnologias digitais para executar os processos de negócios de uma empresa, que incluem tanto as atividades relacionadas com a gestão interna da empresa como com sua coordenação com fornecedores e parceiros do negócio. A modalidade mais conhecida e utilizada de negócios eletrônicos é o comércio eletrônico (e-commerce). Essa posição de destaque faz com que existam muitas pesquisas e ferramentas para a avaliação dessa modalidade que, devido ao seu avanço, pode contribuir para o objeto desta pesquisa. 
Como esta pesquisa se propõe a analisar serviços, vale destacar os estudos realizados por Parasuraman, Zeithaml e Berry (1985), onde se verificou que os consumidores usam critérios similares para avaliar a qualidade do serviço, independentemente do tipo deste serviço. Os autores alocaram esses critérios em dez categorias denominadas "determinantes da qualidade do serviço" que são: confiabilidade, responsabilidade, competência, acesso, cortesia, comunicação, credibilidade, segurança, conhecimento do cliente e tangibilidade. Essas determinantes serviram de base para uma escala denominada SERVQUAL, desenvolvida pelos próprios autores, utilizada para realizar a comparação entre as expectativas dos clientes e suas reais percepções de desempenho.

Hernon e Calvert (2005) desenvolveram um instrumento de pesquisa baseado no SERVQUAL, denominado e-SERVQUAL, que se concentra no exame dos serviços de biblioteca on-line. Com isso, descobriu-se que as dimensões mais importantes para os usuários de um site se encontram na sua facilidade de uso, coleções, confiabilidade, customização/personalização, segurança/privacidade, apoio, facilidade de acesso, articulação, flexibilidade e estética. De acordo com os estudos realizados por Yang, Jun e Peterson (2004), as dimensões de serviços online, centram-se em três dimensões de qualidade: capacidade de resposta, confiabilidade e cortesia. Em função disso, Parasuraman, Zeithaml e Malhotra (2005) desenvolveram uma escala para medir a qualidade dos serviços oferecidos por site, denominada E-S-QUAL, composta pelas dimensões de eficiência, realização, disponibilidade do sistema e privacidade.

Ainda em relação aos avanços alcançados no campo do comércio eletrônico, é possível afirmar que os aspectos funcionais relacionados ao marketing podem trazer importantes contribuições para a avaliação do SRV e, portanto, serão agregados ao modelo proposto neste estudo. Deste modo, torna-se oportuno que os conceitos e os instrumentos de mensuração relacionados a este assunto sejam abordados. As diferenças essenciais entre o contexto físico e o on-line têm levado à reavaliação dos princípios de marketing. Para Constantinides (2002), o tradicional mix de marketing composto pelos 4 Ps (product, price, promotion e place), é incompatível com o contexto eletrônico, e deve ser substituído pelo mix de marketing para web composto pelos 4 Ss: escopo (Scope), Site, Sinergia (Synergy) e 


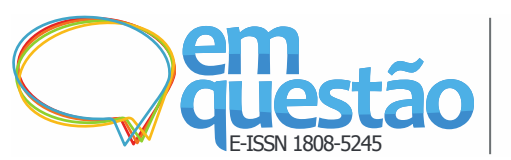

Sistema (System). Para o autor, deve-se considerar a experiência na web, e a impressão que o consumidor tem da empresa on-line - impressão que compreende elementos como pesquisar, navegar, encontrar, selecionar, comparar e avaliar informações, bem como interagir e transacionar com a organização on-line. A experiência na web é essencial, tanto para sites de produtos/serviços quanto para sites focados em conteúdo informativo. Assim, esta experiência é fundamental para os sites que fornecem SRV.

Segundo Constantinides (2004), existem três fatores que influenciam a experiência na web (FIEW) e que são associados ao sucesso ou ao fracasso dos sites: fatores de funcionalidade, psicológicos e de conteúdo que compreende o mix de marketing. Os instrumentos de avaliação aqui apresentados podem servir como um meio para que as organizações analisarem a satisfação de seus usuários. Uma decorrência desse aspecto poderia ser a maior utilização do SRV. É nesse sentido que se coloca o tópico a seguir, que discute a importância da satisfação dos usuários com este tipo de serviço.

\subsection{Satisfação do usuário com o serviço de referência virtual}

Carvalho e Lucas (2005) afirmaram que tanto no Serviço de Referência tradicional quanto no virtual, a satisfação das necessidades de informação dos usuários ocorre por meio da antecipação das respostas através da atualidade e variedade de produtos e serviços oferecidos. As percepções e as necessidades dos clientes são, também para a Reference and User Services Association (2015), importantes medidas de qualidade que impactam os serviços de referência.

$\mathrm{O}$ intuito de oferecer um serviço de qualidade em um meio virtual é fazer com que os usuários fiquem satisfeitos e sintam-se cada vez mais atraídos a utilizálo. Para Parker e Mathews (2001), a satisfação pode ser definida como o sentimento resultante do julgamento que o usuário faz da avaliação do que foi recebido em comparação ao que era esperado, ou seja, a satisfação é o resultado da avaliação da diferença entre as percepções do desempenho e as expectativas.

Devaraj, Fan e Kohli (2002) realizaram uma pesquisa para identificar os antecedentes da satisfação e da preferência do canal que realiza transações do tipo 


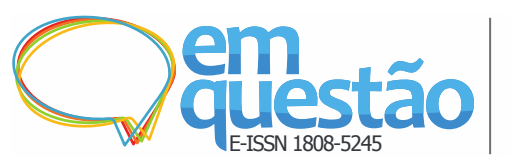

Empresa-a-Consumidores (B2C), e verificaram que a utilidade percebida, a facilidade de uso, a economia de tempo, o preço e a confiabilidade são fatores que afetam a satisfação do canal B2C, e a consequente preferência por ele. Para determinar a satisfação do usuário, Nilsen (2006) coloca que é necessário que fatores como comunicação interpessoal, qualidade das fontes, correção das respostas e eficácia do sucesso do sistema sejam avaliados com frequência. Por meio de um estudo para analisar o índice de satisfação de usuários de serviços de referência presenciais e virtuais, o autor pôde identificar que elementos como o comportamento dos funcionários, links escondidos a esses serviços, solicitação de informações pessoais que não são solicitadas na referência presencial, falta de descrição ou instrução do serviço, ademais de barreiras técnicas, constituem os principais obstáculos para a utilização dos serviços de referência virtual (NILSEN, 2006).

A satisfação dos usuários também foi utilizada por Shachaf, Oltmann e Horowitz (2008), que concluíram que o comportamento positivo ou negativo do membro da equipe de referência, como observado pelo usuário, torna-se um fator significativo no sucesso ou fracasso percebido.

Com a intenção de conhecer o grau de satisfação e a opinião dos utilizadores sobre os serviços prestados pela Biblioteca do Conhecimento Online (b-on), Costa (2007) desenvolveu um questionário eletrônico baseado na escala ServQual e eServQual, que permitiu a obtenção de dados importantes que possibilitaram a definição de linhas de ação que devem ser adotadas no futuro.

Cubillos (2005) destacou a importância de considerar a experiência do usuário também como uma medida de sua satisfação: ao medir o grau de satisfação, mede-se, na verdade, a efetividade, a eficiência e o nível de satisfação do sistema de interface. Pomerantz (2008) ressalta que a avaliação é um componente crítico da gestão de SRV, uma vez que é necessario que haja conhecimento dos pontos fortes e fracos dos serviços existentes para que estes possam ser melhorados. Ainda afirma que o serviço não existe sem seus usuários, de modo que as percepções do atendimento dos usuários são uma parte crítica de qualquer avaliação.

Uma vez que o conceito de satisfação esta intrinsicamente relacionado ao conceito de qualidade, o próximo item deste estudo discorre sobre a importante 
influência que estes conceitos exercem sobre as avaliações que os usuários fazem dos SRVs.

\subsection{Qualidade e satisfação}

Como as bibliotecas se concentram no fornecimento de serviços, Hernon e Calvert (2005) reforçam a importância de abordar a qualidade do serviço: a interação da biblioteca com seus usuários afeta a qualidade e natureza do serviço prestado. Desta forma, é importante procurar atender e/ou superar as expectativas dos usuários, considerando que estes mudam ao longo do tempo, e que suas expectativas são moldadas por experiências com outros prestadores de serviços.

Para Valls e Vergueiro (2006), a finalidade da qualidade é atenuar a diferença entre o que o serviço de informação realmente é e o que o usuário espera deste serviço, sendo que o usuário é o mais indicado para definir suas necessidades e o nível de qualidade do serviço recebido. Os principais requisitos para a qualidade de um serviço de informação são destacados por Vergueiro e Carvalho (2001), que apontam o entendimento das necessidades e expectativas dos usuários; a segurança, que inclui confiabilidade, cortesia e comunicabilidade; e a adoção de linguagem adequada por parte dos profissionais de informação.

Para Parasuraman (2000), os benefícios decorrentes da utilização do ambiente on-line são flexibilidade, conveniência, eficiência e prazer; e os aspectos negativos deste ambiente podem ser segurança, risco de obsolescência, impessoalização e falta de controle.

Madu e Madu (2002), com base em uma aprofundada revisão da literatura, propuseram 15 dimensões da qualidade do serviço on-line: desempenho; apresentação; estrutura; estética; confiabilidade; capacidade de armazenamento; de manutenção; segurança e integridade do sistema; confiança; capacidade de resposta; diferenciação de produtos e customização; políticas da loja Web; reputação; garantia; e empatia.

Já Yang, Peterson e Cai (2003) utilizaram 14 dimensões: resposta, credibilidade, facilidade de uso, confiabilidade, conveniência, comunicação, acesso, 
competência, cortesia, personalização, melhoria contínua, colaboração, segurança/privacidade e estética.

Com o intuito de identificar as dimensões que mais influenciam a qualidade de serviço on-line, Yang, Jun e Peterson (2004) desenvolveram uma pesquisa por meio da qual foi possível identificar seis dimensões, sendo estas a confiabilidade, capacidade de resposta, competência, facilidade de uso, segurança e portfólio de produtos.

Hernon e Calvert (2005) concluíram que, apesar da exploração em torno da qualidade do serviço, não existe um consenso sobre as dimensões a serem exploradas. Por meio de uma extensa revisão da literatura relacionada a qualidade dos serviços eletrônicos, os autores chegaram a dez dimensões: facilidade de uso; estética do site; link; coleções; confiabilidade; suporte; segurança/privacidade/confiança; facilidade de acesso; flexibilidade; e personalização/customização.

Loiacono, Watson e Goodhue (2007) desenvolveram um instrumento para a avaliação de consumidores de sites denominado WebQual, composto por 12 dimensões: tarefa informativa, informações adaptadas, confiança, tempo de resposta, facilidade de compreensão, operações intuitivas, apelo visual, capacidade de inovação, apelo emocional, imagem consistente, completude on-line e vantagem relativa. Outro aspecto norteador utilizado no desenvolvimento deste estudo foi a afirmação de Yang, Jun e Peterson (2004) de que nem todos os atributos de qualidade de serviço têm o mesmo impacto sobre as percepções dos consumidores de serviços on-line. Assim, a identificação dos fatores que mais contribuem para a percepção de qualidade do serviço on-line precisa ser desenvolvida.

Pomerantz e Luo (2006) desenvolveram um estudo para avaliar a eficácia do serviço de referência via chat por meio da satisfação do usuário, e concluíram que avaliar os serviços de referência da perspectiva do usuário e explorar seus feedbacks, constitui uma solução para medir o valor e a utilidade das informações fornecidas por tal serviço aos usuários.

Com o objetivo de explorar maneiras de atenuar a lacuna entre a qualidade do SRV e seu baixo consumo em bibliotecas universitárias, Mu et al. (2011) desenvolveu uma pesquisa onde verificou que a chave do sucesso do SRV é 
preencher a lacuna entre o usuário e o bibliotecário, ao invés de entre o usuário e o serviço. Para o autor, o usuário precisa ter claro que um bibliotecário real está sentado na outra extremidade da linha de bate-papo, e realmente quer que o usuário entre em contato com ele.

\subsection{Modelo para análise dos serviços de referência virtuais}

Os aspectos citados pelos autores nos itens anteriores deste trabalho foram agrupados em sete dimensões: Acesso; Capacidade de Resposta; Comunicação; Confiabilidade; Estímulo; Produto; e Segurança. Para a definição destas dimensões foram consideradas as pesquisas desenvolvidas por diversos autores conforme descrito no quadro um e também no contexto desta pesquisa que diz respeito a avaliação do SRV do ponto de vista de seus usuários.

Quadro 1 - Dimensões do modelo proposto

\begin{tabular}{|c|c|}
\hline Dimensão & Autores \\
\hline Acesso & $\begin{array}{l}\text { Parasuraman, Zeithaml e Berry (1985); Devaraj, Fan e Kohli (2002); Madu } \\
\text { e Madu (2002); Yang, Peterson e Cai (2003); Constantinides (2004); } \\
\text { Parasuraman, Zeithaml e Malhotra (2005); Hernon e Calvert (2005); } \\
\text { Nilsen (2006); Pomerantz e Luo (2006); Loiacono, Watson e Goodhue } \\
\text { (2007); Costa (2007) }\end{array}$ \\
\hline $\begin{array}{l}\text { Capacidade } \\
\text { Resposta }\end{array}$ & $\begin{array}{l}\text { Parasuraman, Zeithaml e Berry (1985); Vergueiro e Carvalho (2001); } \\
\text { Madu e Madu (2002); Yang, Jun e Peterson (2004); Parasuraman, } \\
\text { Zeithaml e Malhotra (2005); Cubillos (2005); Nilsen (2006); Loiacono, } \\
\text { Watson e Goodhue (2007); Connaway, Radford e Dickey (2008) }\end{array}$ \\
\hline Comunicação & $\begin{array}{l}\text { Parasuraman, Zeithaml e Berry (1985); Vergueiro e Carvalho (2001); } \\
\text { Constantinides (2002); Madu e Madu (2002); Yang, Peterson e Cai (2003); } \\
\text { Yang, Jun e Peterson (2004); Constantinides (2004); Parasuraman, } \\
\text { Zeithaml e Malhotra (2005); Hernon e Calvert (2005); Nilsen (2006); } \\
\text { Pomerantz e Luo (2006); Loiacono, Watson e Goodhue (2007); Mu et al. } \\
\text { (2011) }\end{array}$ \\
\hline Confiabilidade & $\begin{array}{l}\text { Parasuraman, Zeithaml e Berry (1985); Vergueiro e Carvalho (2001); } \\
\text { Devaraj, Fan e Kohli (2002); Madu e Madu (2002); Yang, Peterson e Cai } \\
\text { (2003); Constantinides (2004); Yang, Jun e Peterson (2004); Hernon e } \\
\text { Calvert (2005); Loiacono, Watson e Goodhue (2007); Connaway, Radford }\end{array}$ \\
\hline
\end{tabular}




\begin{tabular}{|l|l|}
\hline Estímulo & e Dickey (2008); Shachaf, Oltmann e Horowitz (2008); Costa (2007) \\
\hline Produto & $\begin{array}{l}\text { Constantinides (2002); Madu e Madu (2002); Devaraj, Fan e Kohli (2002); } \\
\text { Yang, Peterson e Cai (2003); Constantinides (2004); Hernon e Calvert } \\
\text { (2005); Nilsen (2006); Loiacono, Watson e Goodhue (2007) }\end{array}$ \\
\hline Segurança & $\begin{array}{l}\text { Yang, Peterson e Cai (2003); Constantinides (2004); Yang, Jun e Peterson } \\
\text { (2004); Hernon e Calvert (2005); Nilsen (2006); Loiacono, Watson e } \\
\text { Goodhue (2007); Shachaf, Oltmann e Horowitz (2008); Costa (2007) }\end{array}$ \\
\hline $\begin{array}{l}\text { Parasuraman, Zeithaml e Berry (1985); Vergueiro e Carvalho (2001); } \\
\text { Madu e Madu (2002); Yang, Peterson e Cai (2003); Yang, Jun e Peterson } \\
\text { (2004); Hernon e Calvert (2005); Parasuraman, Zeithaml e Malhotra } \\
\text { (2005); Nilsen (2006); Costa (2007) }\end{array}$ \\
\hline
\end{tabular}

Fonte: Elaborado pela autora.

Os aspectos que serão avaliados em cada dimensão podem ser visualizados no quadro dois.

Quadro 2 - Aspectos a serem avaliados

\begin{tabular}{|c|c|}
\hline Dimensão & Aspectos a serem analisados \\
\hline Acesso & $\begin{array}{l}\text { - Sempre encontro os materiais eletrônicos que necessito. } \\
\text { - Os links funcionam. } \\
\text { - Écíl navegar pelo site. } \\
\text { - } \quad \text { Disponilmente localizo os serviços disponibilizados. } \\
\text { compreender como informação é organizada. }\end{array}$ \\
\hline $\begin{array}{l}\text { Capacidade de } \\
\text { Resposta }\end{array}$ & $\begin{array}{l}\text { - } \quad \text { Meus problemas são resolvidos rapidamente. } \\
\text { - } \quad \text { Minhas questões são respondidas rapidamente. } \\
\text { - Os funcionários prestam assistência especializada quando preciso. }\end{array}$ \\
\hline Comunicação & $\begin{array}{l}\text { - A interação ocorre de maneira cortês. } \\
\text { - Posso entrar em contato com os bibliotecários a qualquer momento. } \\
\text { - A comunicação através de bate-papo na internet ou de mensagens de texto } \\
\text { - é eficaz. } \\
\text { - Forneço feedback sobre a minha satisfação com o atendimento recebido. }\end{array}$ \\
\hline Confiabilidade & $\begin{array}{l}\text { - Percebo que a biblioteca fornece uma melhoria contínua no atendimento } \\
\text { ao usuário. } \\
\text { - Tenho ajuda e suporte técnico. } \\
\text { - O } \quad \text { sinhas estratégias de busca são registradas e posso usá-las novamente. } \\
\text { - }\end{array}$ \\
\hline Estímulo & $\begin{array}{l}\text { - } \quad \text { Economizo tempo. } \\
\text { - Posso acessá-los nos horários que me são mais convenientes. } \\
\text { - } \quad \text { Esso fazer download de materiais rapidamente. } \\
\text { - } \quad \text { Eu pretendo utilizar esses serviços novamente. } \\
\end{array}$ \\
\hline Produto & - Tenho acesso a uma gama de recursos na minha área especifica. \\
\hline
\end{tabular}




\begin{tabular}{|l|ll|}
\hline & $\begin{array}{l}\text { Tenho acesso a conteúdos eletrônicos atualizados. } \\
\text { • }\end{array}$ & $\begin{array}{l}\text { Recebo alerta sobre o material recém-publicado com base em um perfil de } \\
\text { usuário personalizado. }\end{array}$ \\
& $\bullet \quad$ Tenho acesso as informações on-line claras e de fácil compreensão. \\
\hline Segurança & $\bullet \quad$ O site é seguro. \\
& $\bullet \quad$ Minhas transações são precisamente registradas. \\
& $\bullet \quad$ Me agrada manter o anonimato. \\
\hline
\end{tabular}

Fonte: Elaborado pela autora.

Conforme pode ser observado no Quadro 02, o que se pretende analisar diz respeito aos aspectos funcionais da avaliação de SRV, envolvendo elementos da qualidade do serviço, do mix de marketing e da experiência web do ponto de vista do usuário. Partindo desta premissa, alguns aspectos ressaltados pelos autores pesquisados não serão contemplados por estarem fora do contexto da pesquisa, como é o caso de dois fatores do modelo dos 4 Ss - escopo e sistema -, por exemplo.

\section{Metodologia}

Inicialmente, foi realizado um estudo de caráter bibliográfico com o objetivo de ampliar os conhecimentos sobre os conceitos trabalhados por meio de bases de dados. Tais bases foram consultadas para a busca de artigos de referência, como a Base de Dados Referencial de Artigos e Periódicos em Ciência da Informação (BRAPCI) e Scientific Electronic Library Online (Scielo), além de importantes organizações como Reference and User Services Associantion (RUSA).

Na sequência, realizou-se uma pesquisa quantitativa com seleção de amostra não probabilística por conveniência. A pesquisa por conveniência é uma técnica de abordagem que, de acordo com Malhotra (2009), tem a intenção de obter uma amostra de elementos convenientes cuja seleção fica a cargo do entrevistador. Essa abordagem possibilitou à pesquisadora uma maior compreensão do fenômeno, com a ressalva do fato de que os resultados obtidos apresentam restrições a uma generalização ampla. A análise quantitativa utilizada neste estudo pode ser definida, segundo Richardson (2004), como um método que se utiliza de parâmetros quantificáveis tanto para a coleta dos dados quanto para seu tratamento, e se desenvolve por meio da utilização de técnicas estatísticas. 
Um questionário estruturado foi utilizado para coletar os dados, uma vez que este instrumento enquanto técnica de pesquisa cumpre as funções de descrever as características e medir determinadas variáveis de um grupo social, conforme afirmou Richardson (2004).

Os questionários com afirmações utilizando escala de Likert de cinco pontos foram enviados a usuários de SRVs por meio eletrônico (e-mails). Com a intenção de aumentar a taxa de retorno dos questionários e ajudar uma instituição, um valor simbólico de R \$ 0,30 foi doado ao Grupo de Apoio a Criança com Câncer para cada questionário respondido. Esta doação foi fundamentada em Synodinos (2003), que afirmou que incentivos monetários, que incluem doações para uma instituição de caridade, podem aumentar a taxa de resposta.

A primeira parte do questionário foi construída com o objetivo de caracterizar o entrevistado, mediante questões que permitissem classificar os respondentes por faixa etária, sexo e principal atividade desempenhada. Para a elaboração das demais afirmações, foram utilizados os aspectos relacionados às dimensões que compõem o modelo proposto para analisar a qualidade dos SRVs.

O questionário é composto por 29 afirmações relacionadas às dimensões do modelo proposto neste estudo, sendo que a última afirmação diz respeito à satisfação global do usuário: "Minha nota para minha satisfação em relação a esses serviços é (nota de 1 a 5)".

\section{Resultados}

O convite para responder ao questionário e contribuir com a pesquisa foi desenvolvido e enviado pela internet para cerca de 30.000 e-mails de um banco de dados de usuários pertencentes ao contexto acadêmico. Deste montante, foram obtidas 1.487 respostas, ou seja, 4,96\% dos e-mails enviados retornaram. Não houve necessidade de descartar nenhuma resposta por algum problema de preenchimento, de modo que a amostra a ser considerada neste estudo é composta por 1.487 casos válidos.

Os dados coletados nesta etapa foram inseridos no software de análise estatística SPSS (Statistical Package for Social Sciences) para a aplicação de 
técnicas estatísticas multivariadas que, de acordo com Hair Jr. et al. (2007), são métodos estatísticos que analisam de modo simultâneo várias medidas sobre o objeto da pesquisa. O SPSS é, conforme Landau e Everitt (2004), um pacote de programas para manipular, analisar e apresentar dados, amplamente utilizado nas ciências sociais e comportamentais.

Primeiramente, foi realizada uma análise descritiva da amostra com o objetivo de obter o perfil da mesma. O quadro três apresenta as estatísticas descritivas de soma das notas, média e desvio-padrão das afirmações pesquisadas.

Quadro 3 - Estatística descritiva dos dados obtidos

\begin{tabular}{|c|c|c|c|}
\hline Questões & $\begin{array}{c}\text { Soma das } \\
\text { Notas }\end{array}$ & Média & $\begin{array}{l}\text { Desvio- } \\
\text { Padrão }\end{array}$ \\
\hline Posso acessá-los nos horários que me são mais convenientes. & 6819 & 4,59 & 0,82 \\
\hline Economizo tempo. & 6208 & 4,17 & 1,097 \\
\hline Eu pretendo utilizar esses serviços novamente. & 6152 & 4,14 & 1,066 \\
\hline O site é seguro. & 5826 & 3,92 & 1,019 \\
\hline Tenho acesso a conteúdos eletrônicos atualizados. & 5814 & 3,91 & 1,018 \\
\hline Tenho acesso a uma gama de recursos na minha área especifica. & 5769 & 3,88 & 1,075 \\
\hline A interação ocorre de maneira cortês. & 5739 & 3,86 & 1,083 \\
\hline $\begin{array}{l}\text { Tenho acesso as informações on-line claras e de fácil } \\
\text { compreensão. }\end{array}$ & 5541 & 3,73 & 1,015 \\
\hline Os funcionários prestam assistência especializada quando preciso. & 5523 & 3,71 & 1,138 \\
\hline Os links funcionam. & 5515 & 3,71 & 1,150 \\
\hline Me agrada manter o anonimato. & 5438 & 3,66 & 1,301 \\
\hline
\end{tabular}




\begin{tabular}{|c|c|c|c|}
\hline $\mathrm{O}$ site funciona bem. & 5416 & 3,64 & 1,008 \\
\hline Posso fazer download de materiais rapidamente. & 5297 & 3,56 & 1,224 \\
\hline É fácil navegar pelo site. & 5151 & 3,46 & 1,141 \\
\hline Minhas questões são respondidas rapidamente. & 5064 & 3,41 & 1,206 \\
\hline Minhas transações são precisamente registradas. & 5007 & 3,37 & 1,217 \\
\hline Sempre encontro os materiais eletrônicos que necessito. & 5005 & 3,37 & 1,123 \\
\hline Meus problemas são resolvidos rapidamente. & 4989 & 3,36 & 1,134 \\
\hline $\begin{array}{l}\text { Percebo que a biblioteca fornece uma melhoria contínua no } \\
\text { atendimento ao usuário. }\end{array}$ & 4866 & 3,27 & 1,120 \\
\hline Facilmente localizo os serviços disponibilizados. & 4762 & 3,20 & 1,102 \\
\hline $\begin{array}{l}\text { Disponho de site bem estruturado, com menus que me ajudam a } \\
\text { compreender como informação é organizada. }\end{array}$ & 4756 & 3,20 & 1,170 \\
\hline $\begin{array}{l}\text { Posso entrar em contato com os bibliotecários a qualquer } \\
\text { momento. }\end{array}$ & 4755 & 3,20 & 1,273 \\
\hline $\begin{array}{l}\text { Minhas estratégias de busca são registradas e posso usá-las } \\
\text { novamente. }\end{array}$ & 4645 & 3,12 & 1,268 \\
\hline Tenho ajuda e suporte técnico. & 4438 & 2,98 & 1,148 \\
\hline $\begin{array}{l}\text { A comunicação através de bate-papo na internet ou de mensagens } \\
\text { de texto é eficaz. }\end{array}$ & 4275 & 2,87 & 1,254 \\
\hline $\begin{array}{l}\text { Recebo alerta sobre o material recém-publicado com base em um } \\
\text { perfil de usuário personalizado. }\end{array}$ & 3843 & 2,58 & 1,347 \\
\hline Estes serviços são amplamente divulgados. & 3763 & 2,53 & 1,184 \\
\hline
\end{tabular}


Forneço feedback sobre a minha satisfação com o atendimento recebido.

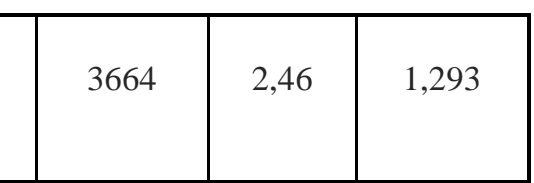

Fonte: Elaborado pela autora.

Conforme os dados apresentados no Quadro 3, verificou-se que das seis dimensões utilizadas no modelo deste estudo, três têm seus atributos dentre os mais bem avaliados, que são as dimensões Estimulo, Segurança e Produto. Vale destacar que dos cinco aspectos mais bem avaliados, três compõem a dimensão Estímulo, demonstrando que os aspectos e recursos disponíveis para estimular a utilização do meio eletrônico por parte dos usuários dos SRVS estão produzindo efeitos positivos.

Em relação às questões com as menores pontuações atribuídas pelos usuários dos SRVs, quatro das seis dimensões utilizadas no modelo deste estudo estão presentes: Confiabilidade, Comunicação, Produto e Estímulo. Dos cinco aspectos com as menores pontuações, dois compõem a dimensão Comunicação. Esta constatação demonstra que os usuários atribuem grande importância à comunicação usuário-bibliotecário, de modo que este deve ser um dos aspectos que deve receber atenção especial por parte dos gestores dos SRVs.

A partir das médias das pontuações alcançadas é possível concluir que, de modo geral, os usuários dos SRVS se mostram satisfeitos com os atributos aqui pesquisados.

Após a análise descritiva, foi realizada uma análise de correlação entre todos os 28 atributos pesquisados que, de acordo com Stevenson (2001), tem como objetivo determinar a força do relacionamento entre duas observações emparelhadas, indicando até que ponto os valores de uma variável estão relacionados com os de outra. Não foram encontrado indícios de correlação entre nenhum dos atributos pesquisados.

Para verificar qual a influência dos atributos pesquisados na satisfação dos usuários de SRVs, foi realizada uma análise de regressão multivariada. A análise de regressão multivariada é uma técnica estatística que pode ser usada para analisar a relação entre uma variável - denominada "dependente" - e uma ou mais outras variáveis - denominadas como "independentes" (HAIR JR. et al., 2007). Assim, utilizou-se esta técnica de dependência com o objetivo de verificar quais variáveis 
possuem influência sobre a variável dependente - no caso, a satisfação dos usuários de SRVs. Em relação ao tamanho da amostra, Hair Jr. et al. (2007) sugerem que a proporção ideal seria de dez respostas para cada variável independente a ser analisada. Esse número foi respeitado e superado.

O Quadro 4 demonstra os modelos encontrados para a equação de satisfação dos usuários elaborados por meio da análise estatística de regressão multivariada.

Quadro 4 - Modelos encontrados por meio da análise de regressão

\begin{tabular}{|c|c|c|c|c|c|c|c|c|c|}
\hline \multirow[b]{2}{*}{ Modelo } & \multirow[b]{2}{*}{$\mathbf{R}$} & \multirow[b]{2}{*}{$\begin{array}{l}\text { R } \\
\text { Quadrado }\end{array}$} & \multirow{2}{*}{$\begin{array}{l}\text { R } \\
\text { Quadrado } \\
\text { Ajustado }\end{array}$} & \multirow[b]{2}{*}{$\begin{array}{l}\text { Erro Padrão } \\
\text { de Coeficiente }\end{array}$} & \multicolumn{5}{|c|}{ Estatísticas Alteradas } \\
\hline & & & & & \begin{tabular}{|l} 
R Quadrado \\
Alterado
\end{tabular} & F Alterado & df1 & df2 & $\begin{array}{l}\text { Sig. F } \\
\text { Alterado }\end{array}$ \\
\hline 1 &, $382^{\mathrm{a}}$ &, 146 &, 145 &, 839 & 0146 & 253,466 & 1 & 1485 &, 000 \\
\hline 2 &, $446^{\mathrm{b}}$ &, 199 &, 198 &, 813 & 0,53 & 98,085 & 1 & 1484 &, 000 \\
\hline 3 &, $474^{\mathrm{c}}$ &, 225 &, 223 &, 800 & 0,26 & 49,422 & 1 & 1483 &, 000 \\
\hline 4 &, $487^{\mathrm{d}}$ & 237 & 235 & ,794 & 0,12 & 24,135 & 1 & 1482 &, 000 \\
\hline 5 &, $496^{\mathrm{e}}$ &, 246 & 243 & ,789 & ,009 & 16,921 & 1 & 1481 &, 000 \\
\hline 6 &, $501^{\mathrm{f}}$ &, 251 &, 248 & ,787 &, 006 & 11,511 & 1 & 1480 &, 001 \\
\hline 7 &, $506^{\mathrm{g}}$ & 256 &, 253 & ,784 &, 005 & 9,272 & 1 & 1479 &, 002 \\
\hline 8 &, $510^{\mathrm{h}}$ &, 260 &, 256 & ,782 &, 004 & 8,572 & 1 & 1478 &, 003 \\
\hline 9 &, $513^{\mathrm{i}}$ &, 263 & 259 & ,781 &, 003 & 5,689 & 1 & 1477 &, 017 \\
\hline
\end{tabular}

a. Preditores: (Constante), Q24

b. Preditores: (Constante), Q24, Q17

c. Preditores: (Constante), Q24, Q17, Q22

d. Preditores: (Constante), Q24, Q17, Q22, Q19

e. Preditores: (Constante), Q24, Q17, Q22, Q19, Q02

f. Preditores: (Constante), Q24, Q17, Q22, Q19, Q02, Q05

g. Preditores: (Constante), Q24, Q17, Q22, Q19, Q02, Q05, Q23

h. Preditores: (Constante), Q24, Q17, Q22, Q19, Q02, Q05, Q23, Q15

i. Preditores: (Constante), Q24, Q17, Q22, Q19, Q02, Q05, Q23, Q15, Q27

j. Variável Dependente: Q29

Fonte: Elaborado pela autora. 
Dentre os modelos gerados mediante a análise de regressão multivariada, foi escolhido o modelo sete, baseando-se no $\mathrm{R}^{2}$ ajustado e na análise do ganho explicativo da mesma. A importância de analisar o $\mathrm{R}^{2}$ ajustado pode ser constatada quando Hair Jr. et al. (2007) salientam que, embora a adição de variáveis independentes sempre faça com que os coeficientes de determinação aumentem, o $\mathrm{R}^{2}$ pode cair se as variáveis independentes acrescentadas tiverem pouco poder de explicação e/ou se os graus de liberdade se tornarem muito pequenos.

Assim, de acordo com os resultados obtidos por meio da análise de regressão multivariada dos dados, a satisfação geral dos usuários com os SRVs pode ser definida mediante a seguinte equação:

SATU $=1,252+0,219 \mathrm{FUNC}+$ 0,137 $\mathrm{INCL}+0,093 \mathrm{ETBU}+0,068 \mathrm{STES}+$ 0,075 CTEL + 0,056 QTRP + 0,055 CMOL

Em que:

SATU = Satisfação dos usuários de serviços de referência virtual;

$\mathrm{FUNC}=\mathrm{O}$ site funciona bem;

INCL $=$ Tenho acesso às informações on-line claras e de fácil compreensão;

ETBU = Minhas estratégias de busca são registradas e posso usa-las novamente;

STES = Disponho de site bem estruturado, com menus que me ajudam a compreender como a informação é organizada;

CTEL $=$ Tenho acesso a conteúdos eletrônicos atualizados;

QTRP = Minhas questões são respondidas rapidamente;

$\mathrm{CMOL}=\mathrm{A}$ comunicação através de bate-papo na internet ou de mensagens de texto é eficaz.

Dos sete atributos considerados na equação de satisfação dos usuários de SRVs, dois compõem a dimensão Confiabilidade (“O site funciona bem” e "Minhas estratégias de busca são registradas e posso usá-las novamente”), dois fazem parte da dimensão Produto ("Tenho acesso às informações on-line claras e de fácil compreensão" e "Tenho acesso a conteúdos eletrônicos atualizados"), um integra a dimensão Acesso ("Disponho de site bem estruturado, com menus que me ajudam a compreender como a informação é organizada"), outro ainda compõe a dimensão Capacidade de Resposta ("Minhas questões são respondidas rapidamente") e, por 
fim, um integra a dimensão Comunicação (“A comunicação através de bate-papo na internet ou de mensagens de texto é eficaz").

$\mathrm{O}$ atributo que representou maior peso na equação de satisfação dos usuários foi “O site funciona bem”, da dimensão Confiabilidade, demonstrando a importância de oferecer um serviço em um meio - no caso eletrônico - que não comprometa a qualidade do serviço disponibilizado. A importância da funcionalidade adequada de um site já havia sido ressaltada por Hernon e Calvert (2005). Outro atributo da dimensão Confiabilidade que também faz parte da composição da equação de satisfação dos usuários de SRVs é "Minhas estratégias de busca são registradas e posso usa-las novamente”. A posição de destaque dada aos atributos relacionados à confiabilidade se faz presente deste os determinantes do SERVQUAL de Parasuraman, Zeithaml e Berry (1985).

O segundo atributo em pontuação na equação de satisfação do usuário foi "Tenho acesso às informações on-line claras e de fácil compreensão", da dimensão Produto. A importância deste atributo está em oferecer o que o usuário deseja de modo claro e fácil para que o mesmo seja motivado a utilizar o site com frequência, bem como recomendá-lo a novos usuários como destacado por Yang, Jun e Peterson (2004). A dimensão Produto também foi representada pelo atributo "Tenho acesso a conteúdos eletrônicos atualizados", na equação de satisfação obtida neste estudo. A atualização das fontes de informações virtuais é uma das vantagens competitivas do SRVs ressaltada por Carvalho e Lucas (2005).

$\mathrm{O}$ atributo "Disponho de site bem estruturado, com menus que me ajudam a compreender como a informação é organizada", da dimensão Acesso, demonstra sua importância ao participar da composição da equação de satisfação do usuário. A estrutura da informação, conforme Constantinides (2004), além de influenciar a experiência na web, melhora a usabilidade do site. Segundo diversos autores, como Vergueiro e Carvalho (2001) e Madu e Madu (2002), a estrutura em que a informação é disponibilizada aos seus usuários é um aspecto importante que influencia na satisfação destes usuários.

A dimensão Capacidade de Resposta também participa da equação de satisfação do usuário por meio do atributo "Minhas questões são respondidas rapidamente". Se o tempo de espera por parte do usuário for demasiado, a agilidade 
do serviço é anulada. Yang, Jun e Peterson (2004) consideraram o tempo de resposta como uma das dimensões de qualidade dos serviços prestados. A satisfação do usuário, para Nilsen (2006), é influenciada pelas respostas recebidas pelos usuarios destes serviços. Outros trabalhos que também destacaram a importância do atendimento das questões de referência em tempo hábil foram Madu e Madu (2002), Pessoa e Cunha (2007) e Accart (2012). Para completar a equação de satisfação do usuário, tem-se o atributo "A comunicação através de bate-papo na internet ou de mensagens de texto é eficaz" da dimensão Comunicação. A importância da comunicação foi destacada por vários autores como Santana et al. (2010), Constantinides (2002), entre outros.

A segurança oferecida por um site é fator crítico de sucesso discutido por importantes autores como Parasuraman, Zeithaml e Berry (1985) que a tem como um de seus "determinantes da qualidade do serviço". Vergueiro e Carvalho (2001), por sua vez, consideraram a segurança como um dos principais requisitos para a qualidade de um serviço de informação, enquanto Constantinides (2004) coloca a confiança online como aspecto influenciador da experiência na web. Hernon e Calvert (2005) abordam em suas pesquisas aspectos de segurança como privacidade e confiança, ao passo que Parasuraman, Zeithaml e Malhotra (2005) desenvolveram o modelo E-S-QUAL, composto também pela dimensão Privacidade, que verifica em que grau o site é seguro e protege as informações do cliente, e Accart (2012) discorre sobre a proteção dos dados. De acordo com a pesquisa realizada neste estudo, é possível concluir que os sites realmente têm se preocupado em oferecer um ambiente seguro aos seus usuários, considerando que o atributo relacionado à segurança do site estava entre os cinco atributos que obtiveram, na análise descritiva, as maiores pontuações.

Para que os SRVs possam ser implantados com sucesso, autores como Silva e Beuttenmüller (2005) e Connaway, Radford e Dickey (2008) ressaltam a importância de se desenvolver estratégias de marketing adequadas para que os usuários possam tomar conhecimento das ofertas de SRVs e, assim, além de utilizálos, promovê-los a potenciais usuários. Para os autores, estas estratégias ainda ocorrem de modo insuficiente. $\mathrm{O}$ atributo relacionado à divulgação dos SRVs estava entre os que receberam as piores pontuações na pesquisa, ressaltando a carência da 
divulgação destes serviços. Apesar da insuficiência de estratégias de marketing que pudessem proporcionar a adequada divulgação dos SRVS, os resultados obtidos demonstraram que os usuários pretendem utilizar os SRVs novamente. A afirmação correspondente a utilizar os SRVs estava entre as que obtiveram as maiores pontuações durante a realização da pesquisa.

A comunicação on-line é um atributo importante destacado por vários autores, como Pessoa e Cunha (2007), Parasuraman, Zeithaml e Berry (1985), e Constantinides (2004). A eficácia da comunicação através de bate-papo na internet ou de mensagens de texto alcançou papel de destaque ao compor a equação de satisfação dos usuários de SRVs apresentada neste estudo, porém, o fato de estar entre as afirmações que receberam as piores pontuações na pesquisa é algo que deve chamar atenção, demonstrando que, apesar de sua importância, é um atributo que ainda deve ser aprimorado.

Pomerantz e Luo (2006) afirmaram a importância de explorar os feedbacks dos usuários que utilizam os serviços. Vergueiro e Carvalho (2001) também fizeram importantes considerações sobre a avaliação do usuário sobre o serviço. Apesar da importância destacada, os resultados alcançados neste estudo demonstram que solicitar e fazer uso de feedbacks de usuários parece ser algo que não acontece com frequência, uma vez que o atributo relacionado ao fornecimento de feedbacks estava entre os que receberam as piores pontuações.

\section{Considerações finais}

Com o objetivo de propor um modelo para analisar os SRVs do ponto de vista de seus usuários, e com o intuito de que este modelo pudesse ser testado, optou-se pela realização uma pesquisa quantitativa por meio de aplicação de questionários em meio eletrônico. O principal ponto a ser destacado é que o modelo proposto se mostrou um instrumento adequado para analisar os SRVs, de fácil aplicação e que pode se adequar a diversas situações. 
Com a aplicação do modelo, importantes pontos foram destacados:

a) a necessidade de avaliar os SRVs com mais frequência por meio da solicitação de feedbacks aos seus usuários;

b) a falta de estratégias de marketing para a adequada divulgação dos SRVs;

c) a carência de serviços personalizados.

As principais contribuições deste estudo para a área acadêmica estão relacionadas com o desenvolvimento de um modelo que permita analisar os SRVs, e que agregue, de modo inovador e complementar, aspectos considerados por importantes estudos realizados nesta área, considerando o ponto de vista do usuário dos serviços avaliados.

Em relação à área gerencial, é possível afirmar que este estudo contribuiu com o desenvolvimento de um modelo de fácil aplicação para analisar SRVs sob a perspectiva de seus usuários, auxiliando a identificação de aspectos importantes para os usuários destes serviços e que poderiam não estar recebendo a devida atenção de seus gestores.

Considerando que o SRV se trata de um assunto dinâmico e multidisciplinar que pode envolver áreas tão distintas e abrangentes, seria impossível, em um único estudo, cobrir de maneira eficiente todos os seus aspectos. Portanto, o estudo em questão teve seu foco nos serviços prestados aos usuários, ciente de que outros elementos devem ser estudados em pesquisas futuras.

\section{Referências}

ACCART, J-P. Serviço de referência: do presencial ao virtual. Brasília: Briquet de Lemos, 2012.

ARELlANO, M. A. M. Serviços de referencia virtual. Ciência da Informação, Brasília, v. 30, n. 2, p. 7-15, maio/ago. 2001.

CARVALHO, L. S.; LUCAS, E. R. O. Serviço de referência e informação: do tradicional ao on-line. In: CINFORM - ENCONTRO NACIONAL DE CIÊNCIA DA INFORMAÇÃO, 6., 2005, Salvador. Anais... Salvador: [s.n.], 2005.

CONNAWAY, L. S.; RADFORD, M. L.; DICKEY, T. J. Virtual reference services: on the trail of the elusive non-user: what research in virtual reference environments 
reveals. Bulletin of the American Society for Information Science and Technology, Silver Spring, v. 34, n. 2, p. 25-28, Dec./Jan. 2008.

CONSTANTINIDES, E. Influencing the online consumer's behavior: the Web experience. Internet Research, Bingley, v. 14, n. 2, p. 111-126, 2004.

CONSTANTINIDES, E. The 4S web-marketing mix model. Electronic Commerce Research and Applications, Miamisburg, v. 1, n. 1, p. 57-76, Spring 2002.

COSTA, M. T. Biblioteca do conhecimento online: pela construção da sociedade do conhecimento. Actas dos Congressos de Bibliotecários, Arquivistas e Documentalistas, Lisboa, n. 9, mar. 2007. Disponível em: <http://www.apbad.pt/Downloads/congresso9/COM3_.pdf>. Acesso em: 06 maio 2014.

CUBILLOS, M. F. La satisfacción del usuario remoto de la biblioteca. Biblios, Brasília, ano 6, n. 21-22, enero/agosto 2005.

DEVARAJ, S.; FAN, M.; KOHLI, R. Antecedents of B2C channel satisfaction and preference: validating e-commerce metrics. Information Systems Research, Catonsville, v. 13, n. 3, p. 316-333, Sept. 2002.

HAIR JR., J. F. et al. Análise multivariada de dados. 5. ed. Porto Alegre: Bookman, 2007.

HERNON, P.; CALVERT, P. E-service quality in libraries: exploring its features and dimensions. Library \& Information Science Research, Philadelphia, v. 27, n. 3, p. 377-404, Summer 2005.

KATZ, B. Digital reference: an overview. The Reference Librarian, Philadelphia, v. 38, n.79-80, p. 1-17, 2002.

LANDAU, S.; EVERITT, B. S. A handbook of statistical analyses using SPSS. Boca Raton: Chapman \& Hall/CRC, 2004.

LAUDON, K. C.; LAUDON, J. P. Sistemas de informação gerenciais. 9. ed. São Paulo: Pearson Prentice Hall, 2010.

LOIACONO, E. T.; WATSON, R. T.; GOODHUE, D. L. WebQual: an instrument for consumer evaluation of web sites. International Journal of Electronic Commerce, Ann Arbor, v. 11, n. 3, p. 51-87, Spring 2007.

MADU, C. N.; MADU, A. A. Dimensions of e-quality. International Journal of Quality \& Reliability Management, Bingley, v. 19, n. 3, p. 246-258, 2002.

MALHOTRA, N. K. Marketing research: an applied orientation. 6th ed. Harlow: Prentice Hall, 2009. 
McNEAL, R. S. et al. Innovating in digital government in the American States. Social Science Quarterly, Hoboken, v. 84, n. 1, p. 52-70, Mar. 2003.

MU, X. et al. A survey and empirical study of virtual reference service in academic libraries. The Journal of Academic Librarianship, Orlando, v. 37, n. 2, p. 120129, Mar. 2011.

NILSEN, K. Comparing users' perspectives of in-person and virtual reference. New Library World, Bingley, v. 107, n. 1222/1223, p. 91-104, 2006.

PARASURAMAN, A. Technology readiness index (TRI): a multiple-item scale to measure readiness to embrace new technologies. Journal of Service Research, Newcastle upon Tyne, v. 2, n. 4, p. 307-320, May 2000.

PARASURAMAN, A.; ZEITHAML, V. A.; BERRY, L. L. A conceptual model of service quality and its implications for future research. Journal of Marketing, Birmingham, v. 49, n. 4, p.41-50, Autumn 1985.

PARASURAMAN, A.; ZEITHAML, V. A.; MALHOTRA, A. E-S-QUAL: a multiple-item scale for assessing electronic service quality. Journal of Service Research, Newcastle upon Tyne, v. 7, n. 3, p. 213-233, Feb. 2005.

PARKER, C.; MATHEWS, B. P. Customer satisfaction: contrasting academic and consumers' interpretations. Marketing Intelligence \& Planning, Bingley, v. 19, n. 1, p. 38-44, 2001.

PEREIRA, M. R. S.; BRENHA, T. C. P. Serviço de referência em bibliotecas reflexões. In: CONGRESSO BRASILEIRO DE BIBLIOTECONOMIA, DOCUMENTAÇÃO E CIÊNCIA DA INFORMAÇÃO SISTEMAS DE INFORMAÇÃO, MULTICULTURALIDADE E INCLUSÃO SOCIAL, 24., 2011, Maceió. Anais... Maceió: [s.n.], 2011.

PESSOA, P.; CUNHA, M. B. Perspectivas dos serviços de referência digital. Informação \& Sociedade: Estudos, João Pessoa, v. 17, n. 3, p. 69-82, set./dez. 2007.

POMERANTZ, J. Evaluation of online reference services. Bulletin of the American Society for Information Science and Technology, Silver Spring, v. 34, n. 2, p. 15-19, Dec./Jan. 2008.

POMERANTZ, J.; LUO, L. Motivations and uses: Evaluating virtual reference service from the users' perspective. Library \& Information Science Research, Philadelphia, v. 28, n. 3, p. 350-373, Autumn 2006.

REFERENCE AND USER SERVICES ASSOCIATION. Guidelines for implementing and maintaining virtual reference services. Chicago: RUSA, 2004. 
REFERENCE AND USER SERVICES ASSOCIATION. Measuring and assessing reference services and resources: a guide. Chicago: RUSA, [2015?]. Disponível em:

$<$ http://www.ala.org/rusa/sections/rss/rsssection/rsscomm/evaluationofref/measrefgu ide>. Acesso em: 23 fev. 2015.

RICHARDSON, R. J. Metodologia da pesquisa aplicável às ciências sociais. In: BEUREN, I. M. (Org.). Como elaborar trabalhos monográficos em contabilidade: teoria e prática. 2. ed. São Paulo: Atlas, 2004.

SANTANA, Anderson et al. Atendimento online em bibliotecas: a experiência da universidade de São Paulo. In: SEMINARIO NACIONAL DE BIBLIOTECAS UNIVERSITARIAS, 16., 2010, Rio de Janeiro. Trabalhos... Rio de Janeiro: UFRJ, 2010. Disponível em: <http://www.ip.usp.br/portal/images/stories/atendimentoonline-bibliotecas>. Acesso em: 08 maio 2014.

SHACHAF, P.; OLTMANN, S. M.; HOROWITZ, S. M. Service equality in virtual reference. Journal of the American Society for Information Science and Technology, Hoboken, v. 59, n. 4, p. 535-550, Feb. 2008.

SILVA, A. K. A.; BEUTTENMÜLLER, Z. F. O serviço de referência online nas bibliotecas virtuais da região nordeste. Enc. BIBLI: R. eletrônica de Bibl. Ci. Inform., Florianópolis, n. 20, p. 75-91, $2^{\circ}$ semestre de 2005.

SIQUEIRA, J. C. Repensando o serviço de referência: a possibilidade virtual. Pontodeacesso, Salvador, v. 4, n. 2, p. 116-130, set. 2010.

STEVENSON, W. J. Estatística aplicada à administração. São Paulo: Harbra, 2001 .

SYNODINOS, N. E. The "art" of questionnaire construction: some important considerations for manufacturing studies. Integrated Manufacturing Systems, Bingley, v. 14, n. 3, p. 221-237, 2003.

VALLS, V. M.; VERGUEIRO, W. C. S. A gestão da qualidade em serviços de informação no Brasil: uma nova revisão de literatura, de 1997 a 2006. Perspectiva em Ciência da Informação, Belo Horizonte, v. 11, n. 1, p. 118-137, jan./abr. 2006.

VERGUEIRO, W.; CARVALHO, T. de. Definição de indicadores de qualidade: a visão dos administradores e clientes de bibliotecas universitárias. Perspectiva em Ciência da Informação, Belo Horizonte, v. 6, n. 1, p. 27-40, jan./jun. 2001.

YANG, Z.; JUN, M.; PETERSON, R. T. Measuring customer perceived online service quality: scale development and managerial implications. International Journal of Operations \& Production Management, Bingley, v. 24, n. 11, p. 11491174, 2004. 
YANG, Z.; PETERSON, R. T.; CAI, S. Services quality dimensions of Internet retailing: an exploratory analysis. Journal of Services Marketing, Bingley, v. 17, n. 7, p. 685-700, 2003.

\title{
The virtual reference services: quantitative analysis \& model
}

\begin{abstract}
With plenty resources still to be researched, the virtual reference services works around the different ways to provide the reference services through technological information, in benefit of not only the universities' libraries, but also for its digital users. In this context of Universities' Libraries, through the analysis of the tools developed for the digital environment, this paper intends to create a new analysis plan to analyze the virtual reference services from the point of view of its users, aiming to make these services better and more valuable. This plan was used and analyzed along with users of the services offered by universities' libraries, so it could be fully validate, and therefore used as a real tool to measure this kind of service.
\end{abstract}

Keywords: Virtual reference service. Reference service: analysis. User's satisfaction.

Recebido: 23/11/2015

Aceito: 11/04/2016 